between the observed and the calculated intensities was sufficiently good to afford confirmation of the correctness of these values. Interatomic distances calculated from these adopted atomic parameters suggests a regular distribution of the silver and the cadmium atoms, silver atoms occupying positions of $B$ atoms and $C$ atoms, cadmium atoms those of $A$ atoms and $D$ atoms.

Our calculations wore based on powder photographs and the data of reflections made available to us by Prof. A. Westgren of the University of Stockholm. A detailed account of them will be published in the Acta et Commentationes Universitatis Tartuensis.

\section{Harald Perlitz.}

Rolf Aavakivi.

Laboratory of Theoretical Physics, University, Tartu. Sept. 20.

\section{Cataphoretic Measurements on Solutions of Visual Purple and Indicator Yellow}

LIGHT transforms visual purple, the substance responsible for scotopic vision, into a yellow com. pound, the colour of which depends on $p H$ ('indicator yellow'1). Visual purple behaves as a coloured protein, that is, it is a 'chromoprotein'. Experiments have been carried out to seo if a shift of the iso. electric point occurs on bleaching.

The visual purple was obtained from Hungarian frogs by means of extraction with digitonin, and the solution dialysed for long enough to remove tho bulk of the salts, but not to precipitate the protein. Veasurements were made with a micro-cataphoretic cell which will bo described elsewhere. Quartz rar. ticles of the size 2-5 $\mu$ were suspended in the solution and $N / 100$ acetate buffer added. The particles adsorb the chromoprotein and their movement is governed by the ionization of the latter ${ }^{2}$. The measurements on visual purple were carried out in ruby light to provent bleaching. After each determination, whito light was switched on without removing the cell, so transforming the visual purple into indicator yellow. During the bleaching the cataphoretic velocity of the particles increased in

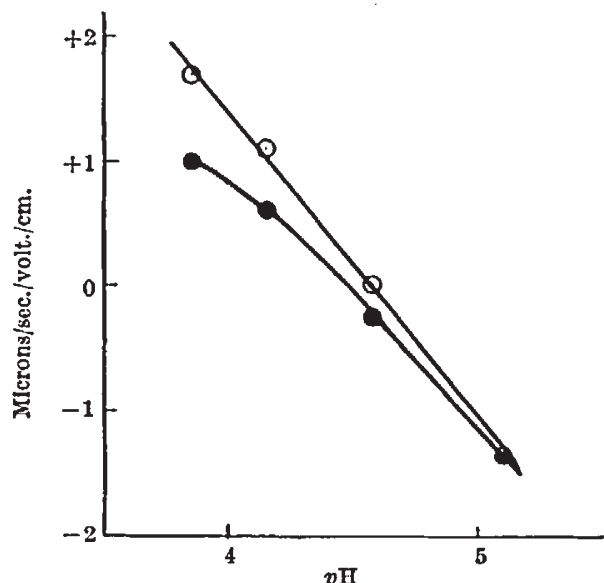

The Variation of the Cataphoretic Velocities IN Solutions of Visual Purple and Indicator Yellow with $p H$ VALUE solutions more acid than about $p H 4 \cdot 5$, and decreased in solutions more alkaline than $p H 4 \cdot 5$. After a few minutes a steady velocity was reached, which was independent of whether observation was made with ruby or white light.

The cat aphoretic velocities beforo and after bleaching for one preparation are plotted against $p H$ in the accompanying graph. It appears that the isoelectric point of the particles in the visual purple solution is 4.47 , and in the indicator yellow solution $4.57 \mathrm{pH}$ units. Other preparations gave the same type of curves and the samo shift on bleaching, but sometimes the positions of the isoelectric points were slightly different $( \pm 0 \cdot 1)$. 'The isoelectric points are, therefore, in a comparatively strongly acid range. This explains the observation that solutions of visual purple become acid on prolonged dialysis against distilled water ${ }^{3}$.

No other caso appears to bo recorded in literature where moderate illumination produces a change in the isoelectric point of a protein or chromoprotein. The bleaching process of visual purple is apparently associated with an increase in the number of alkaline groups, such as $\mathrm{NH}_{2}$, available for ionization.

Our thanks aro duo to the Rockefeller Foundation for financial help.

E. E. Broda.

C. F. Goodrve.

R. J. LYTHGOE.

E. VICTOR.

University College,

London.

Septernber 6.

s Lythgoe, R. J., J. Physiol., 89, 331 (193i)

Abramson, H. A., J. Amer. Chem. Soc., 50, 390 (1928).

I,ythgoe, R. J., and Quilliam, J. P., J. Physiol, 93, 24 (1939).

A Mechanism for the Concentrating of Potassium by Cells, with Experimental Verification for Muscle

THE membrane considered is one of special type, with cation and anion pores, permeable to potassium but not to larger sodium ions, and permeable at the same time to small anions of the type of chloride, bicarbonate, phosphate, etc., but not to larger anions such as hexoso esters, phosphocreatine, etc. The concentrations inside (or within the cell) and outside the membrane are represented as follows: $B / V$ and $b$, impermeable cations; $k_{1}$ and $k$, potassium ; $d_{1}$ and $d$, permeablo anions; $A / V$, impermeable noncolloidal anions; $C / V$, colloidal anions inside; $x$, external impermeable non-electrolyte such as glucose.

The permeable anions, which will bo almost entirely chloride and bicarbonate, aro considered as mono. valent, and the valencies of $B, A$ and $C$ are written as $m, n$ and $p . V$ is tho volume of the solvent inside, which is considered to be initially one litre. The quantities $A, B$ and $C$ aro regarded as independent of $V$. The total external concentration is written as ' $c$ '.

From the requirements of the osmotic, electrical and Donnan equilibria we may deduce the following equations :

$$
\begin{aligned}
& V^{2}\left(c^{2}-4 k d\right)-2 V K c+\left(K^{2}-K_{1}^{2}\right)=0 . \\
& 2 k_{1}=c-\left(K-K_{1}\right) / V \text {. . . . } \\
& k d=k_{1} d_{1}, \quad \text {. . . . . (3) } \\
& \text { where } K=B+A \text {, } \quad \text {. } \quad \text {. } \quad \text {. (4) } \\
& \text { and } K_{1}=n A+p C-m B \text {. . . (5) }
\end{aligned}
$$

Reckoning the value of $\left(K-K_{1}\right)$ from the available data, it is found to be sufficiently small to be neglected 